\title{
The Model of Stochastic Optimization of Production under Uncertainty and Risk
}

\author{
Alex Ivanovich Borodin ${ }^{1} \&$ Nataliya Nikolaevna Shash ${ }^{1}$ \\ ${ }^{1}$ Faculty of Finance, Plekhanov Russian University of Economics, Russian Federation \\ Correspondence: Nataliya Nikolaevna Shash, Plekhanov Russian University of Economics, Stremyanny lane, 36, \\ Moscow, 117997, Russian Federation. E-mail:aib-2004@yandex.ru; nat_vshu@mail.ru
}

Received: December 20, 2017

Accepted: February 27, $2018 \quad$ Online Published: April 19, 2018

doi:10.5539/ass.v14n5p33

URL: https://doi.org/10.5539/ass.v14n5p33

\begin{abstract}
The article describes possibilities of application of models of stochastic optimization of output. The features of the application of various problems of stochastic optimization. The expediency of application of methods of network planning method, PERT, and in the process create the network model of production. It is shown that in conditions of uncertainty and risk when it is difficult to accurately determine the values of the parameters of the problem the use of stochastic models in the planning process of production allows to achieve higher profitability. The authors propose a stochastic model of optimal output and practical recommendations for its application in the activities of industrial enterprises is concluded that to improve the efficiency of planning processes of production it is advantageous to combine the proposed stochastic optimization model with the method of analysis and evaluation of programs.
\end{abstract}

Keywords: stochastic optimization methods, economic-mathematical model, the problem of stochastic optimization, stochastic model, risk, uncertainty, PERT method

\section{Introduction}

In the dynamic external business environment, when the risk becomes a natural and unavoidable factor model stochastic optimization can demonstrate a high efficiency in the process of solving the task of determining the optimal production, inventory levels, investment portfolio. Stochastic formulation of the problem more accurately reflects the economic reality in terms of medium-term planning period. Moreover, the use of stochastic methods significantly exceeds the efficiency of deterministic models in the formation of the optimal production plan, improving financial and economic results of the company's business including profit figures.

It is obvious that the determined characteristics are not suitable for any company in determining the optimal release if you need to increase the planning horizon under conditions of risk and uncertainty. In practice optimization problems with random initial parameters are casual. For example, when planning the activities of industrial enterprises there is always uncertainty associated with the inaccuracy or incompleteness of information on demand, changes in market prices, suppliers and so on.

This is especially true for enterprises with a long production cycle. Random variables include intensity of orders at the enterprises of services industry, number of buyers in shops, number of passengers, etc. Therefore if it is impossible to set the parameter values unambiguously (standards of resources expenses, raw materials stocks, value, etc.) in an applied task, it is referred to as a stochastic one. Methods of task solution with random factors are called the methods of stochastic optimization.

In his studies the author will be guided by rather a detailed analysis of the last achievements and publications which is carried out in the last twenty years (Marti, 2015; Sarker, 2007; Williams, 2013; Song, 2013; Bertocchi, Consigli, \& Michael, 2011; Uryasev \& Pardalos, 2001; Dzemyda, Saltenis, \& Zilinskas, 2002; Zabinsky, 2003; Houmin, George, \& Qing, 2006). This source contains the general statements of problems of stochastic optimization, which can serve as the beginning of scientific development.

Common problem here is the absence of concrete practical recommendations what types of probabilistic distributions are to be used in one case or another. Often the sufficient economic justification isn't given to the offered approaches and the mathematics in them is used formally.

The purpose of the article is to develop the model of optimum production in stochastic statement. The author 
plans to offer methods of the task realization, having analysed the model on sensitivity to the parameter changes of a production system.

\section{Method}

In mathematical methods of the operations research such direction as stochastic programming is defined. We will pay attention to methods and models of this direction.

Existence of statistical information allows to evaluate selective characteristics of socio-economic systems: empirical function of probabilities distribution, mathematical expectation, dispersion, etc. It is natural that such problems are solved in the conditions of uncertainty and risk. They can be divided into one-stage, two-stage and multi-stage ones (Ali, Khompatraporn, \& Zabinsky, 2005).

One-stage problems of stochastic optimization are the ones where the decision is made once and doesn't change any more. Such approach is often implemented to optimally place the production capacities.

In two-stage tasks the initial decision can be modified at the second stage of management of the socio-economic system. For example, the enterprise has no unambiguous information on demand for the new production. Therefore at the first stage production batch allowing to estimate demand is issued. The finishing (second) stage on the basis of information from the first stage forms the modified optimum plan of release.

In two-stage tasks the initial decision can be modified at the second stage of management of social and economic system. For example, the enterprise has no unambiguous information on demand for the new production. Therefore at the first stage a sample quantity is issued allowing to estimate the demand. The finishing (second) stage forms the modified optimum plan of output on the basis of information from the first stage.

Multi-stage problems allow any number of the correction activities. This refers to problems of stockpile management in consecutive periods, optimization of investment projects, operational management of production, technological and other processes.

Problems of stochastic optimization are various in many aspects. For example, if violation of some restrictions leads to serious consequences, it is a task of so-called rigid statement. Restrictions can have probabilistic character. The researcher in stochastic programming might face numerous difficulties regarding the interpretation of probabilistic characteristics, the choice of the efficiency criterion, etc.

In the determined case the researcher isolates the optimum decision from a set of admissible decisions which comply with all restrictions of a task. By stochastic optimization it is possible to consider to be the admissible decision the one which does not comply in an insignificant measure with some of restrictions. Such statement complicates a problem and therefore the system of penalties for violation of restrictions is implemented.

\section{Results}

Stochastic models may contain casual coefficients in criterion function and casual components in the system of task restrictions. We will consider the most widespread statements of problems of stochastic programming.

We will assume that coefficients of the criterion function $\mathrm{Cj}(\mathrm{j}=1, \mathrm{n})$ are random variables, i.e. $\mathrm{Cj}(\omega)$. Other parameters of the model are considered to be determined.

We will discuss, whether such an approach will be reasonable. For this purpose we will consider a classical problem of optimum production.

Let the enterprise start producing $n$ new items of the products $\mathrm{P}_{1}, \mathrm{P}_{2}, \ldots, \mathrm{P}_{\mathrm{n}} . M$ resources $\mathrm{S}_{1}, \mathrm{~S}_{2}, \ldots, \mathrm{S}_{\mathrm{m}}$ are used for the production by which we will mean raw materials, materials, components, etc. $\mathrm{B} i$ resources $(\mathrm{i}=1, \mathrm{~m})$ are stocked in the warehouse. $\mathrm{A}_{\mathrm{ij}}$ units the resource $\mathrm{S} i$ are spent for the production of $\mathrm{P} j$. All the listed parameters are determined.

Table 1. Problem of optimum production in stochastic statement

\begin{tabular}{cccccc}
\hline Resources & \multicolumn{2}{c}{ Resource consumption for a production unit } & \multicolumn{2}{c}{ Resource supplies } \\
& $P_{1}$ & $P_{2}$ & $\ldots$ & Pn & \\
\hline$S_{1}$ & $a_{11}$ & $a_{12}$ & $\ldots$ & $a_{1 n}$ & $b_{1}$ \\
$S_{2}$ & $a_{21}$ & $a_{22}$ & $\ldots$ & $a_{2 n}$ & $b_{2}$ \\
$\ldots$ & $\ldots$ & $\ldots$ & $\ldots$ & $\ldots$ & $\ldots$ \\
$S_{m}$ & $a_{m 1}$ & $a_{m 2}$ & $\ldots$ & $a_{m n}$ & $b_{m}$ \\
\hline Price of a production unit & $c_{1}(\omega)$ & $c_{2}(\omega)$ & $\ldots$ & $c_{n}(\omega)$ & \\
Quantity of the output & $\mathrm{x}_{1}$ & $\mathrm{x}_{2}$ & $\ldots$ & $\mathrm{x}_{\Pi}$ & \\
\hline
\end{tabular}


Since products arrive to consumers for the first time, their market price is supposed to be random. We will designate these prices through $\mathrm{Cj}(\omega)(\mathrm{j}=1, \mathrm{n})$. The data referring to such a problem of linear programming is placed in Table 1.

Let $Z(\omega)$ (mon. unit) be the sales revenue. The stochastic problem definition can be reduced to the determined case if we take the mathematical expectation from the criterion function. The mathematical model of a problem of linear programming composed according to table 1 will look as follows:

$$
\begin{gathered}
\mathrm{M}[\mathrm{Z}(\omega)]=\sum_{j=1}^{n} M\left[C_{j}(\omega)\right] X_{j} \rightarrow \max , \\
\sum_{j=1}^{n} a_{i j} x_{j} \leq b_{j}(i=1, m), \\
x_{j} \geq 0(j=1, n) .
\end{gathered}
$$

Adequacy of model will considerably depend on the choice of probabilities distribution of random variables $\mathrm{Cj}$ $(\omega)(j=1, n)$. Since it is about prices for products, these are the continuous random variables possessing the values from the corresponding intervals.

The author suggests using the random variables which are evenly distributed on a segment $[\alpha ; \beta]$. According to the task the ends of the segment can be only positive numbers.

To determine such random variables, it is necessary to define the distribution segments. It can be done using statistical methods. Having let out a sample quantity of products $\mathrm{P} j$, we will determine by selection the smallest $\alpha j$ and the largest $\beta j$ prices. Having done the same thing with other goods, we will find out that random variables $\mathrm{C} j(\omega)$ are distributed evenly on segments $[\alpha j ; \beta j](\mathrm{j}=1, \mathrm{n})$.

The mathematical expectation of the evenly distributed random variable $C j(\omega)$ is calculated as $[\alpha j ; \beta j]$. Having defined $Z^{\prime} d e f=\mathrm{M}[\mathrm{Z}(\omega)]$, we will come to such a criterion function:

$$
Z^{\prime}=\sum_{j=1}^{n} \frac{\alpha_{j}+\beta_{j}}{2} x_{j} \rightarrow \max
$$

Such approach allows to apply methods of linear optimization in the determined form. Along with the initial task

we will consider a dual task

$$
\begin{aligned}
& x_{j} \geq 0 \quad(j=1, n), \\
& \left\{\begin{array}{l}
a_{11} x_{1}+a_{12} x_{2}+\cdots+a_{1 n} x_{n} \leq b_{1} \\
a_{21} x_{1}+a_{22} x_{2}+\cdots+a_{2 n} x_{n} \leq b_{2} \\
\cdots \cdots \cdots \cdots \cdots \cdots \cdots \cdots \cdots \cdots \cdots \cdots \cdots \cdots \cdots \cdots \cdots \cdots \cdots \cdots \cdots \\
a_{m 1} x_{1}+a_{m 2} x_{2}+\cdots+a_{m n} x_{n} \leq b_{m} \\
\mathrm{Z}=c_{1} x_{1}+c_{2} x_{2}+\cdots+c_{n} x_{n} \rightarrow \max
\end{array}\right.
\end{aligned}
$$

$$
\begin{gathered}
y_{i} \geq 0 \quad(j=1, n), \\
a_{11} y_{1}+a_{12} x y_{2}+\cdots+y_{m 1} x_{m} \geq c_{1} \\
a_{21} y_{1}+a_{22} y_{2}+\cdots+a_{m 2} y_{m} \geq c_{2} \\
\cdots \cdots \cdots \cdots \cdots \cdots \cdots \cdots \cdots \cdots \cdots \cdots \cdots \cdots \cdots \cdots \cdots \cdots \cdots \cdots \cdots \cdots \\
a_{1 n} y_{1}+a_{2 n} y_{2}+\cdots+a_{m n} y_{m} \geq c_{n} \\
\mathrm{~F}=b_{1} y_{1}+b_{2} y_{2}+\cdots+b_{m} y_{m} \rightarrow \text { min. }
\end{gathered}
$$

According to the first duality theorem if one of problems of a dual couple has the optimum solution, and other task has the optimum solution, and extreme values of criterion functions coincide:

$$
\sum_{j=1}^{n} c_{j} x_{j}^{*}=\sum_{i=1}^{m} b_{i} y_{i}^{*}
$$


If criterion function of one of tasks isn't limited, thearea of feasible solutions of other task is empty.

The formation of a dual task enables us to carry out the economic-mathematical analysis of the initial problem of linear optimization.

We will consider the initial problem of optimum production in a matrix-vector form:

$$
\begin{gathered}
Z=\vec{C}^{*} \vec{X} \rightarrow \text { max }, \\
\overrightarrow{A X} \leq \vec{B} \\
\vec{X} \geq 0
\end{gathered}
$$

Here $\mathrm{A}$ is a matrix from coefficients at unknowns in the limitation system; $\mathrm{B}^{\rightarrow}$ is a vector of resources supplies; $\vec{C}$ is a vector of the product prices; $\vec{X}$ is the required plan of production which will maximize the revenue from realization of $Z$.

Let $\vec{Y}=\left(\mathrm{y}_{1}, \mathrm{y}_{2} \ldots, \mathrm{y}_{\mathrm{m}}\right)$ be the solution to the dual task which has to minimize costs of resources F. Having introduced the transposed matrix $A^{T}$, we will write down the dual task in a matrix-vector form:

$$
\begin{gathered}
\mathrm{F}=\overrightarrow{B^{*}} \vec{Y} \rightarrow \min , \\
\mathrm{A}^{\mathrm{T}} \overrightarrow{\mathrm{Y}} \geq \overrightarrow{\mathrm{C}}, \\
\overrightarrow{\mathrm{Y}} \geq 0
\end{gathered}
$$

According to the second duality theorem, if some variable $x_{j}^{*}(j=1, n)$ of the optimal solution of the original problem is positive, then the $\mathrm{j}$-th restriction of the dual problem of its optimal solution becomes strict equality. If the optimal solution of the original problem turns an $i-$ th $(i=1, m)$ restriction in strict equality, the optimal solution of the dual problem variable $y_{i}^{*}$ is bigger than 0 .

Thus dual estimates are an indication of the scarcity of resources and products. Value $\mathrm{y}_{\mathrm{i}}^{*}$ is called the dual assessment or shadow price of the $i$-th resource. If $y_{i}^{*}>0$, then the resource is scarce and by the implementation of an optimal plan $\overrightarrow{\mathrm{X}}^{*}$ is completely consumed. I.e. the i-th constraint addressyb the original problem in strict equality. Acquisition of an additional unit of this resource will result in an increase in revenue from the sale of the value of $Z$ by the value of $y_{i}^{*}$. The higher the value of the shadow price is, the more scarce the resources are. For a readily available resource $\mathrm{y}_{\mathrm{i}}^{*}=0$.

Further analysis of solving linear optimization is based on a study of the sensitivity of the optimal plan to changes in the values of the parameters $\mathrm{Cj}, \mathrm{a}_{\mathrm{ij}}, \mathrm{bi}(\mathrm{i}=1, \mathrm{~m} ; \mathrm{j}=1, \mathrm{n})$. Therefore, such an analysis is briefly called sensitivity analysis.

Suppose that the initial vector of resource stocks has the form of $\vec{B}=\left(b_{1}, b_{2} \ldots, b_{m}\right)$. Let us introduce the increment $\Delta \mathrm{b}_{\mathrm{i}}$ and the result of such increments $\mathrm{b}_{\mathrm{i}}+\Delta \mathrm{b}_{\mathrm{i}}(\mathrm{i}=1, \mathrm{~m})$. Denoting with $\Delta \overrightarrow{\mathrm{B}}=\left(\Delta \mathrm{b}_{1}, \Delta \mathrm{b}_{2} \ldots, \Delta \mathrm{b}_{\mathrm{m}}\right)$ the increment vector, we obtain a new vector of resource stocks $\vec{B}+\Delta \vec{B}$. Now substitute the $\vec{B}$ vector in the original and dual problems with the $\overrightarrow{\mathrm{B}}+\Delta \overrightarrow{\mathrm{B}}$. Thus a symmetrical pair of dual multiparameter problems will be formed.

This article has already mentioned the first duality theorem. Its main assertion is that $\mathrm{Zmax}=$ Fmin or in another form $\overrightarrow{\mathrm{C}} \cdot \overrightarrow{\mathrm{X}}^{*}=\mathrm{B} \cdot \overrightarrow{\mathrm{Y} *}$, where $\mathrm{Y}$ is a vector of unknown dual problem. We denote with $\overrightarrow{\mathrm{X}}_{\Delta}^{*}$ the vector of the optimal solutions multiparameter problem. By duality we obtain that $\overrightarrow{\mathrm{C}} \cdot \overrightarrow{\mathrm{X}}_{\Delta}^{*}=(\overrightarrow{\mathrm{B}}+\mathrm{AB}) \cdot \mathrm{Y}^{*}$.

Consider the increase of the objective function of the original problem:

$$
\Delta \overrightarrow{\mathrm{Z}} \max =\overrightarrow{\mathrm{C}} \cdot \overrightarrow{\mathrm{X}}_{\Delta}^{*}-\overrightarrow{\mathrm{C}} \cdot \overrightarrow{\mathrm{X}}^{*}=(\overrightarrow{\mathrm{B}}+\mathrm{AB}) \cdot \mathrm{Y}^{*}-\mathrm{B} \cdot \overrightarrow{\mathrm{Y} *}=\Delta \overrightarrow{\mathrm{B}} \cdot \overrightarrow{\mathrm{Y} *}
$$

If we change only the $\mathrm{i}$-th constraint, then $(\Delta \overrightarrow{\mathrm{Z}} \max ) \mathrm{i}=\Delta \mathrm{b}_{\mathrm{i}} \mathrm{y}_{\mathrm{i}}^{*}$. From which we derive that:

$$
\mathrm{y}_{\mathrm{i}}^{*}=\frac{(\Delta \mathrm{Zmax}) \mathrm{i}}{\Delta \mathrm{b}_{\mathrm{i}}} \quad(\mathrm{i}=1, \mathrm{~m}) .
$$

After passing the limit we have:

$$
y_{i}^{*}=\frac{\partial m a x}{\partial b_{i}} \quad(i=1, m) .
$$

Therefore, the dual estimates are an indication of the impact of restrictions on the value of the objective function. 
Therefore, it is of practical interest to calculate the limits of the right-limits $b_{i}$ ( the lower and upper boundaries of resource stocks), in which the optimal plan $\overrightarrow{\mathrm{X}}^{*}$ remains the same.

We fix the basic unknowns included in the optimal plan. Assume that the data have numerical values $\mathrm{x}_{1}^{*}, \mathrm{x}_{2}^{*}, \ldots, \mathrm{x}_{\mathrm{m}}^{*}$. The basic unknown correspond to $\mathrm{m}$ vector-columns of coefficients in the matrix, which is obtained from the matrix A by adding the balance column in the formation of the canonical form of linear programming problem. Let us compose a matrix out of these column vectors

And now let us calculate the inverse matrix

$$
\mathrm{W}=\left[\begin{array}{c}
\mathrm{w}_{11}+\mathrm{w}_{12}+\cdots+\mathrm{w}_{1 \mathrm{~m}} \\
\mathrm{w}_{21}+\mathrm{w}_{22}+\cdots+\mathrm{w}_{2 \mathrm{~m}} \\
\cdots \cdots \cdots \cdots \cdots \cdots \cdots \cdots \\
\mathrm{w}_{\mathrm{m} 1}+\mathrm{w}_{\mathrm{m} 2}+\cdots+\mathrm{w}_{\mathrm{mm}}
\end{array}\right.
$$

$$
W^{-1}=\left\{\begin{array}{c}
d_{11}+d_{12}+\cdots+d_{1 m} \\
d_{21}+d_{22}+\cdots+d_{2 m} \\
\cdots \cdots \cdots \cdots \cdots \cdots \cdots \cdots \cdots \cdots \\
d_{m 1}+d_{m 2}+\cdots+d_{m m}
\end{array}\right.
$$

Dual estimates are used for economic analysis solutions, provided that the resources are changed only within certain limits. The intervals of resource sustainability is given by the following formulas:

$$
\left[b_{i}-\Delta b_{i}^{-} ; b_{i}+\Delta b_{i}^{+}\right] \quad(i=1, m)
$$

where the lower reduction limit $\Delta \mathrm{b}_{\mathrm{i}}^{-}$and the upper reduction limit $\Delta \mathrm{b}_{\mathrm{i}}^{+}$are calculated the following way:

$$
\begin{aligned}
\Delta b_{i}^{-} & =\min _{d j i>0} \frac{x_{j}^{*}}{d j i} \\
\Delta b_{i}^{+} & =\min _{d j i<0} \frac{x_{j}^{*}}{d j i}
\end{aligned}
$$

It is important to note that if the $\mathrm{i}$-th column of the matrix $\mathrm{W}^{-1}$ does not contain negative numbers, and there are only positive and equal to zero numbers, then $\Delta \mathrm{b}_{\mathrm{i}}^{+}$will be taken for $+\infty$.

Dual estimates are an indication of the feasibility of production of new products. Suppose we have the opportunity to start producing $\mathrm{P}_{\mathrm{n}+1}$. Resource consumption rates for the production of one unit are respectively $a_{1, n+1} ; a_{2, n+1} ; \ldots, a_{m, n+1}$. The price per unit is $c_{n+1}$. The expediency of production is determined by the sign of the index:

$$
\Delta_{\mathrm{n}+1}=\mathrm{c}_{\mathrm{n}+1}-\sum_{\mathrm{i}=1}^{\mathrm{m}} \mathrm{a}_{\mathrm{i}, \mathrm{n}+1} \cdot \mathrm{y}_{\mathrm{i}}^{*}
$$

If $\Delta_{\mathrm{n}+1}>0$, then the production is profitable, $\Delta_{\mathrm{n}+1}=0$ - we break even, $\Delta_{\mathrm{n}+1}<0$ - unprofitable.

Dual estimates are also used as a tool for comparing the conventional costs and benefits. If you change the amount of resources within the sustainability impact of the $\mathrm{i}$-th individual resource by the amount of income from sales is defined as $(\Delta \mathrm{Zmax}) \mathrm{i}=\Delta \mathrm{b}_{\mathrm{i}} \mathrm{y}_{\mathrm{i}}^{*}$. If $(\Delta \mathrm{Zmax}) \mathrm{i}>0$, the income will increase by $(\Delta \mathrm{Zmax}) \mathrm{i}$ per unit of account, otherwise it will decrease. The cumulative effect of changes in the number of resources is calculated as follows:

$$
\Delta \mathrm{Zmax}=\Delta \overrightarrow{\mathrm{B}} \cdot \overrightarrow{\mathrm{Y} *}=\sum_{\mathrm{i}=1}^{\mathrm{m}}(\Delta \mathrm{Zmax}) \mathrm{i}
$$

Consider the possibility of additional purchase of the $\mathrm{i}$-th resource in volume $\Delta \mathrm{b}_{i}^{+}$at the price of $\mathrm{p}_{\mathrm{i}}$ per resource unit. The acquisition costs totaled at $\Delta \mathrm{b}_{\mathrm{i}}^{+} \cdot \mathrm{p}_{\mathrm{i}}$. The increment of income will be $\Delta \mathrm{b}_{\mathrm{i}}^{+} \bullet \mathrm{y}_{\mathrm{i}}^{*}$. If the increment of income exceeds the cost of the acquisition, i.e.

$$
\Delta \mathrm{b}_{\mathrm{i}}^{+} \cdot \mathrm{y}_{\mathrm{i}}^{*}-\Delta \mathrm{b}_{\mathrm{i}}^{+} \cdot \mathrm{p}_{\mathrm{i}}>0,
$$

then it is advisable to purchase. Otherwise it is not. 
Stability interval unit price of products is called the $\mathrm{i}$-th interval $\left[\mathrm{C}_{\mathrm{i}}^{\min } ; \mathrm{C}_{\mathrm{i}}^{\max }\right]$ with the following properties. If the price of $\mathrm{Ci} \in\left[\mathrm{C}_{\mathrm{i}}^{\min } ; \mathrm{C}_{\mathrm{i}}^{\max }\right]$, and the prices for other types of production are fixed, the optimal production plan $\overrightarrow{\mathrm{X}}^{*}$ remains unchanged.

In order to determine the stability intervals we need to recall the geometric interpretation of linear programming problems. Supporting plans - this corner points formed by the intersection of the hyperplanes system limitations. In finding the extreme value of the objective function hyperplane-isoobjective with the normality vector $\overrightarrow{\mathrm{C}}=$ $\left(c_{1}, c_{2} \ldots, c_{m}\right)$ moves to the optimal plan $\vec{X}$ etc.

\section{Discussion}

The organization of a modern and high-tech industry requires a large number of calendar linking of interrelated activities. The compilation and analysis of the schedules is a complex task in dealing with which the method of network planning. These methods make it possible to determine the following. First, what works or operations of the many that make up the project are critical of their effect on the overall duration of the project calendar. Second, how to build the best possible schedule of all activities of the project to support the target date at minimal cost.

Under the network model of organization of production we mean the economic-mathematical model that reflects the full range of activities and events related to the project. We describe a method of analysis and evaluation of programs PERT (Program Evaluation and Review Technique). It was proposed for practical purposes in 1958.

The method of analysis and evaluation of programs differs from the deterministic methods that are calculated for each transaction of its probabilistic characteristics. It is used to control the timing of the project. The PERT method is focused on the analysis of such projects, for which the duration of the implementation of all or some of the work is not possible to determine accurately. First of all, it is about the design and implementation of new productions. In such projects, many of the works are unique. As a result, there is uncertainty in the timing of the project as a whole.

The PERT method assumes that the execution time of each operation is a random variable. It is necessary to define the following three estimates: a - optimistic time (performance under the most favorable conditions); $\mathrm{m}$ the most likely time (execution time of normal operation); $b$ - pessimistic time (performance in adverse conditions).

Numerous studies (Catoni and Picard, 2004; Spall, 2004; Cao, 2007; Shukla and Mishra, 2014; Marti, 2015; Williams, 2013; Song, 2013; Bertocchi, Consigli and Michael, 2011) showed that the execution of the work is well described by a beta probability distribution. The expectation (average) time performance can be estimated from the formula:

$$
\mathrm{M}_{\mathrm{t}} \approx \frac{\mathrm{a}+4_{\mathrm{m}}+\mathrm{b}}{6}
$$

The estimated variance equals:

$$
\mathrm{D}_{\mathrm{t}} \approx \frac{\mathrm{b}-\mathrm{a}}{6}
$$

And given the fact that $b \geq a$, we obtain an estimate for the standard deviation of the time of the operation:

$$
\sigma_{\mathrm{t}} \approx \frac{\mathrm{b}-\mathrm{a}}{6}
$$

Suppose $\mathrm{T}$ is the time the project. If the project requires works to be done, the timing performance of which one can only assume that the time $\mathrm{T}$ is a random variable. The expectation of the time of the project $\mathrm{M}[\mathrm{T}]$ is the sum of the expected values of working time Mt, lying on the critical path. A similar assumption is made with respect to the dispersion and $\mathrm{D}[\mathrm{T}]$.

To determine the critical path of the project critical path method is used. At this stage, the analysis of project execution time work is assumed to be the expected time t. E. Mt.

It is expected that the project time $\mathrm{T}$ is the sum of a sufficiently large number of independent, identically distributed random t-variables. Under these conditions, the central limit theorem of probability theory. This means that the random variable $\mathrm{T}$ has asymptotically normal probability distribution with time parameters $\mathrm{M}$ [T] and $\sigma[\mathrm{T}]=\sqrt{\mathrm{D}[\mathrm{T}]}$.

We can set a specific deadline for the project $T_{0}$. Then the probability that the project time $\mathrm{T}$ does not exceed a 
specified period $\mathrm{T}_{0}$, is approximately calculated as follows:

where

$$
\mathrm{P}\left\{\mathrm{T} \leq \mathrm{T}_{0}\right\} \approx \frac{1}{2}+\Phi\left(\frac{\mathrm{T}_{0}-\mathrm{M}[\mathrm{T}]}{\sigma[\mathrm{T}]}\right)
$$

$$
\Phi(\mathrm{z})=\frac{1}{\sqrt{2 \pi}} \int_{0}^{\mathrm{z}} \mathrm{e}^{-\mathrm{x}^{2} / 2} \mathrm{dx}
$$

Despite the prospects of using models of stochastic optimization, it is necessary to note that stochastic methods do not guarantee obtaining exact solutions, but they usually allow you to find close enough for the practical use of the solution in a reasonable time. Stochastic nature of most of the methods makes the application of a non-trivial task, since each algorithmic implementation for each class of optimization problems the efficiency, performance, convergence, the impact of the problem and algorithm parameters require thorough research.

\section{Conclusion}

This article offers a fairly wide range of methods for the optimization of socio-economic systems.

As a practical orientation problems the problem of optimal production is addressed. In order to develop production plans the author proposes a specific model of stochastic optimization. This model provides a detailed feasibility study. Methods for solving such problems are described.

It is proposed to use the model not in isolation, but in conjunction with the method of analysis and evaluation of programs. This method allows to design complex manufacturing systems in conditions of uncertainty.

\section{References}

Ali, M. M., Khompatraporn, Ch., \& Zabinsky, Z. B. (2005). A Numerical Evaluation of Several Stochastic Algorithms on Selected Continuous Global Optimization Test Problems. Journal of Global Optimization, 31(4), 635-672. https://doi.org/10.1007/s10898-004-9972-2

Bertocchi, M., Consigli, G., \& Michael, A. H. (2011). Stochastic Optimization Methods in Finance and Energy: New Financial Products and Energy Market Strategies. Springer.

Cao, X.-R. (2007). Stochastic Learning and Optimization: A Sensitivity-Based Approach. Springer.

Catoni, O., \& Picard, J. (2004). Statistical Learning Theory and Stochastic Optimization. Springer.

Dzemyda, G., Saltenis, V., \& Zilinskas, A. (2002). Stochastic and Global Optimization. Springer.

Houmin, Y., George, Y., \& Qing, Z. (2006). Stochastic Processes, Optimization, and Control Theory: Applications in Financial Engineering, Queueing Networks, and Manufacturing Systems. Springer.

Marti, K. (2015). Stochastic Optimization Methods: Applications in Engineering and Operations. Research Springer. https://doi.org/10.1007/978-3-662-46214-0_1

Sarker, R. A. (2007). Optimization Modelling: A Practical Approach. Publisher: CRC Press.

Shukla, N., \& Mishra, N. (2014). Stochastic modeling and optimization of multiplant capacity planning problem. Hong Kong Polytechnic University. https://doi.org/10.1504/IJIEI.2014.066209

Song, D.-P. (2013). Optimal Control and Optimization of Stochastic Supply Chain. Systems Springer. https://doi.org/10.1007/978-1-4471-4724-4_1

Spall, J. C. (2004). Stochastic Optimization. Copyright Springer Heidelberg.

Sundaram, R. K. (1996). A First Course in Optimization Theory. Cambridge Academ.

Uryasev, S., \& Pardalos, P. M. (2001). Stochastic Optimization: Algorithms and Applications. Springer.

Williams, H. P. (2013). Model Building in Mathematical Programming. Wiley.

Zabinsky, Z. B. (2003). Stochastic Adaptive Search for Global Optimization. Springer. https://doi.org/10.1007/978-1-4419-9182-9

\section{Copyrights}

Copyright for this article is retained by the author(s), with first publication rights granted to the journal.

This is an open-access article distributed under the terms and conditions of the Creative Commons Attribution license (http://creativecommons.org/licenses/by/4.0/). 